\title{
10 Jahre Facharzttitel für Infektiologie FMH
}

Ursula Maria Flückiger ${ }^{a}$ Andreas Widmer ${ }^{b}$

a Prof. Dr. med., Präsidentin der Schweizerischen Gesellschaft für Infektiologie

b Prof. Dr. med., Sekretär der Schweizerischen Gesellschaft für Infektiologie
1 Diamond J. Guns, Germs, and Steel: The Fates of Human Societies. W. W. Norton 1997. ISBN 0-393-03891-2.

Korrespondenz: Prof. Dr. med. Ursula Maria Flückiger Universitätsspital Base Klinik für Infektiologie und Spitalhygiene Petersgraben 4 CH-4031 Basel Tel. 0612652525 Fax 0612653198

uflueckiger@uhbs.ch www.sginf.ch
Die «Schweizerische Gesellschaft für Infektiologie» wurde am 13.11.1990 mit dem Ziel gegründet, Erkenntnisse und Forschung über Infektionskrankheiten $\mathrm{zu}$ fördern und Kriterien für die Weiterbildung aufzustellen, die als Grundlage für den Erwerb eines Facharzttitels FMH für Infektiologie dienen sollen.

Die Schaffung eines Facharztes war zu Beginn umstritten: Ein erstes Gesuch wurde abgelehnt, aber die komplexen Therapieschemata mit neuen antiviralen Substanzen gegen HIV und deren zahlreichen Interaktionen, die Verbreitung von multiresistenten Bakterien wie dem methizillin-resistenten $S$. aureus, multiresistente Tuberkulose, komplexe Fremdkörperinfektionen und neue diagnostische Möglichkeiten durch Einführung der Polymerase-Ketten-Reaktion (PCR) waren Gründe, die Anerkennung des Facharztes zu erreichen.

Prof. Michel Glauser und Prof. Claude Regamey haben in dieser Phase die entscheidende Grundlagenarbeit geleistet, um mit dem damaligen Präsidenten Prof. Werner Zimmerli und Sekretär Prof. Andreas Widmer dem Facharzt zum Durchbruch zu verhelfen. Vor 10 Jahren, also 1999, war es dann so weit und das Fachgebiet Infektiologie erhielt den Facharzttitel FMH. Einzigartig für unsere Gesellschaft ist, dass Pädiater und Adultmediziner gemeinsam vertreten sind.

Fachärzte für Infektiologie sind heute in den meisten Regionen vertreten (Abbildung). Im Spitalbereich befasst sich die Infektiologie vor allem im Konsiliardienst mit der Mitbetreuung bei auftretenden Infektionen nach chirurgischen Eingriffen, bei schweren Infektionen in der Intensivmedizin, bei Patienten nach Transplantationen, bei immunsupprimierten Patienten, bei Patienten mit Fremdkörperinfektionen usw. Wichtig sind interdisziplinäre Besprechungen mit den behandelnden Ärzten betreffend gezielter weiterer Untersuchungen und Therapien. Bei der Diagnostik und Empfindlichkeitstestung gegen Antibiotika steht die enge Zusammenarbeit mit den Mikrobiologen im Vordergrund. Wichtig ist die Zusammenarbeit mit Spitalepidemiologen und die Verhinderung bzw. Behandlung von nosokomialen Infektionen. Ein ebenfalls bedeutender Aspekt der Infektiologie ist die Behandlung von Patienten mit einer HIV-Infektion. Aufgrund der heutigen guten Therapiemöglichkeiten werden HIVInfizierte vor allem ambulant behandelt. Die Schweizerische HIV-Kohortenstudie, eine Langzeitbeobachtungsstudie, an der mit HIV-1 infizierte Menschen teilnehmen, stellt ein qualitativ hochstehendes Instrument sowohl für klinische wie auch für die Grundlagenforschung dar. Sie wurde 1988 gegründet und hat massgeblich zum hohen Behandlungs- und Forschungsstandard beigetragen.

Bei Fragen der Epidemiologie und der Verhütung und Behandlung von Infektionskrankheiten sind die Infektiologen wichtige Partner der Behörden, z. B. des BAG und der Kantonsärzte. Gerade die Verbreitung des Grippevirus H1N1 zeigt, wie eng und gut die Zusammenarbeit zwischen Behörden, Infektiologen, Mikrobiologen und Epidemiologen sein muss, damit eine optimale Versorgung der Bevölkerung gelingt.

Jared Diamond hat in seinem Buch «Guns, Germs, and Steel: The Fates of Human Societies» [1] die wichtigsten Einflüsse auf die Geschicke einer Gesellschaft auf 3 Punkte beschränkt: Die «Germs» oder Mikroorganismen werden auch in Zukunft unser Leben wesentlich beeinflussen und das Ziel der Gesellschaft für Infektiologie bleibt, sich kontinuierlich für Forschung, neue Diagnostik und Therapien einzusetzen.

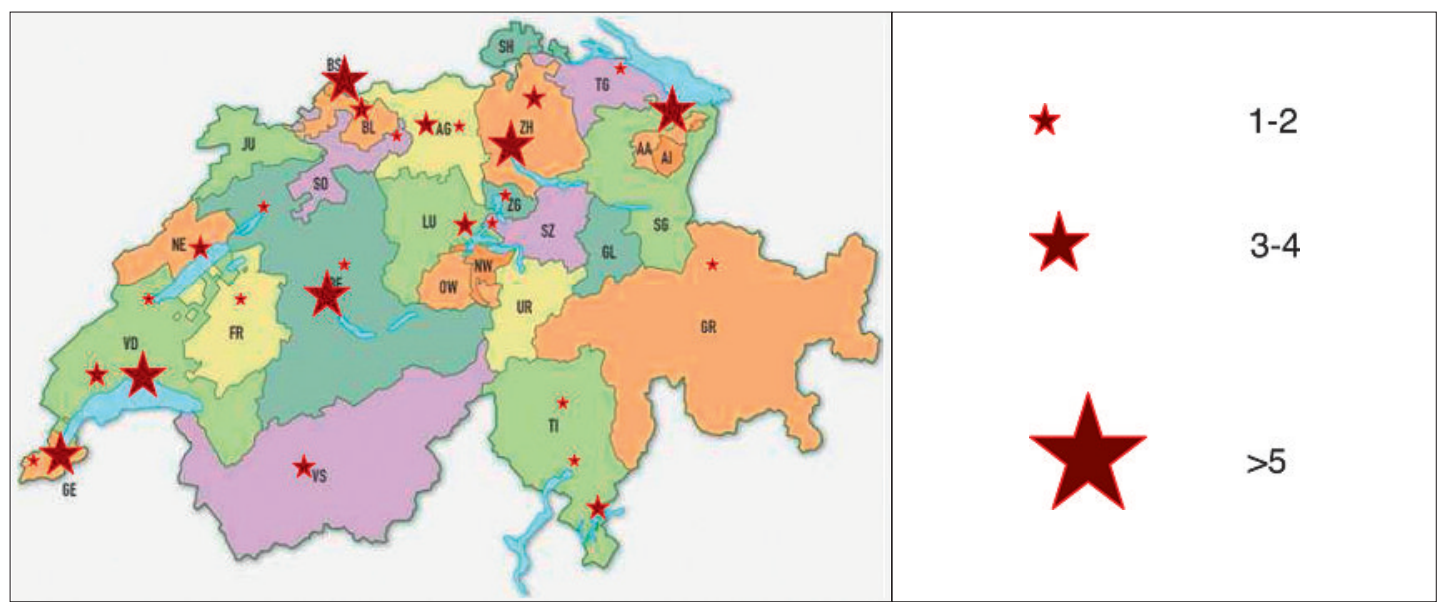

Anzahl Vertreter (ordentliche Mitglieder) der Schweizerischen Gesellschaft für Infektiologie. 